\title{
CASE OF THE MONTH
}

\section{Progressive onset of low back pain: unusual imaging findings on CT and MRI}

\author{
${ }^{1} \mathrm{P}$ F MONTORIOL, MD, ${ }^{2} \mathrm{R}$ BELLINI, MD and ${ }^{2} \mathrm{~J} \mathrm{~L} \mathrm{MICHEL,} \mathrm{MD}$ \\ ${ }^{1}$ Department of Adult Medical Imaging, Estaing University Hospital, Clermont-Ferrand, France, and ${ }^{2}$ Department of \\ Osteoarticular and Thoracic Imaging, Gabriel Montpied University Hospital, Clermont-Ferrand, France
}

Received 18 October 2010 Revised 10 January 2011 Accepted 18 January 2011

DOI: $10.1259 / \mathrm{bjr} / 33368552$

(C) 2011 The British Institute of Radiology

\section{Case report}

A 58-year-old male with a history of chronic hepatitis $\mathrm{C}$ and a known chronic multisystem disease who was under long-term corticosteroid treatment with secondary diabetes presented with progressive onset of a diffuse lower back pain without any associated radiation in the lower limbs. No raised temperature or weight loss was noted.

On standard biological tests, erythrocyte sedimentation rate (ESR) was not elevated and C-reactive protein was normal $\left(1.6 \mathrm{mg} \mathrm{l}^{-1}\right)$. White cell blood count was normal. A mild hyperglycaemia was noted.

No significant abnormalities were found on plain film images of the thoracic and lumbar spine. Given this rather atypical presentation, a MRI examination of the low thoracic and lumbar spine was performed with sagittal short-tau inversion recovery (STIR) (Figure 1), $T_{1}$ weighted (Figure 2) and gadolinium-enhanced $T_{1}$ weighted (Figure 3 ) sequences.

A recent follow-up CT scan of the thorax, abdomen and pelvis was also available and secondarily reformatted in the sagittal plane (Figure 4).

What is your diagnosis? 


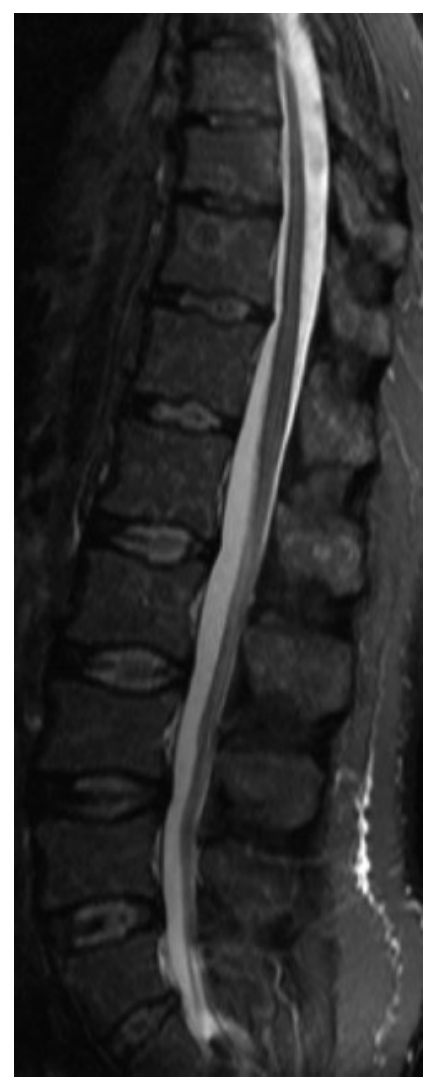

Figure 1. Sagittal short-tau inversion recovery image of the lumbar spine.

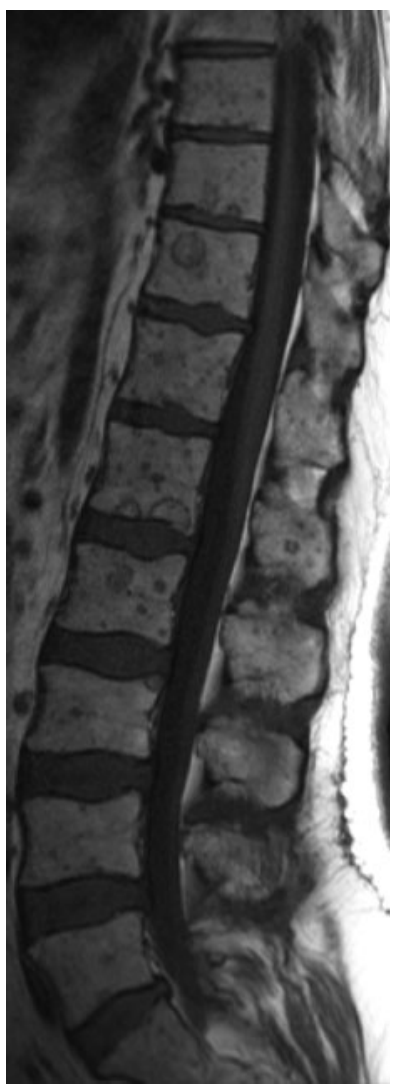

Figure 2. Sagittal $T_{1}$ weighted MRI.

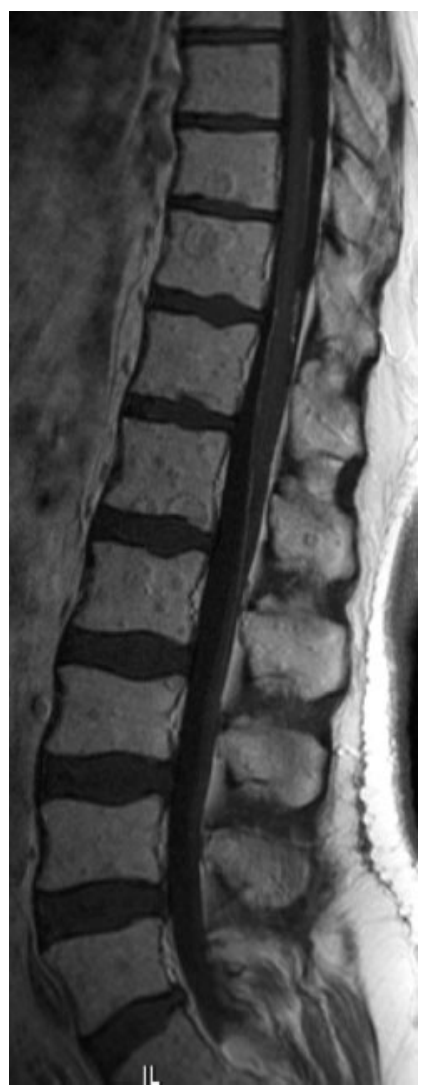

Figure 3. Sagittal $T_{1}$ weighted image after gadolinium administration.

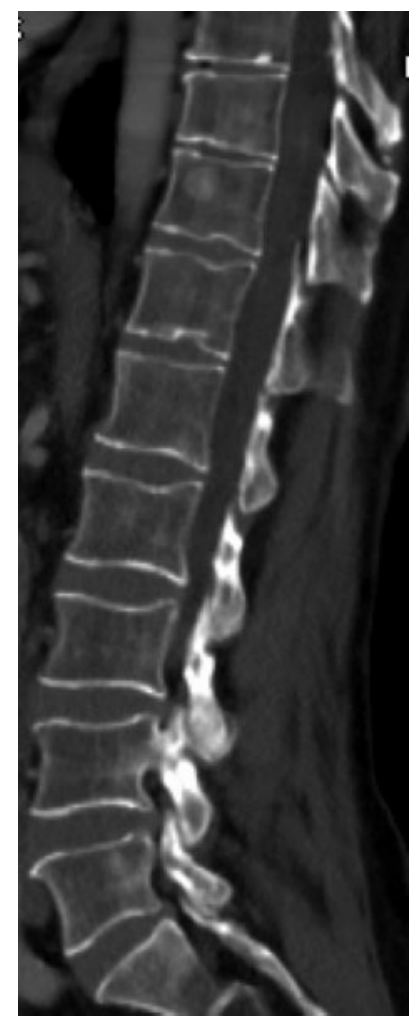

Figure 4. Sagittal reformatted CT scan. 


\section{Diagnosis}

The MRI showed multiple well-delineated nodular lesions within several vertebral bodies.

Most of these lesions appeared similar in signal to fatty bone marrow on $T_{1}$ weighted images and were occasionally relatively lower in signal. The lesions were hypointense with a faint hyperintense rim on STIR, which reflects their fatty nature. Heterogeneous enhancement was seen after administration of gadolinium chelates. The disc spaces were preserved. On CT images these lesions were very slightly osteosclerotic and quite ill-defined. No lytic area was present and the cortical margins were preserved.

The case was discussed at a multidisciplinary team meeting and although the diagnosis of osseous sarcoidosis was probable a decision was made to obtain histological proof. The patient had biopsy-proven history of systemic sarcoidosis, i.e. a mediastinal node 8 years ago (the previously mentioned multisystem disease). He had Stage II disease with bilateral partially calcified mediastinal and hilar nodes associated with diffuse pulmonary infiltrates without fibrosis. The spleen was also affected with multiple small hypoattenuating nodules within an enlarged spleen on CT images.

A CT-guided osseous biopsy of the L1 vertebral body was performed. Histological analysis revealed a gigantocellular non-necrotising granuloma, which is consistent with sarcoidosis. There were no signs of malignancy on the rest of the bone sample and no histological signs of infection, especially tuberculosis.

The patient received higher doses of corticosteroids and showed some improvement in lower back pain. An 18 month clinical and radiological follow-up showed no evolution.

The final diagnosis was diffuse vertebral sarcoidosis.

\section{Discussion}

Sarcoidosis is a chronic granulomatous disease of unknown origin that mostly affects young and middleaged patients. It is characterised by non-caseating granulomas with proliferation of epithelioid cells. Distinct geographical and racial predilections exist with African-Americans, Swedes and Danes more frequently affected. Clinical presentation remains non-specific and may include weight loss, fatigue and sometimes mild fever. Approximately 50\% of the affected patients are asymptomatic [1]. Lungs, lymph nodes and skin are the most commonly affected sites.

Osseous involvement is rare. It occurs in approximately $5-10 \%$ of patients with sarcoidosis [1]. Phalanges of the hands and feet are the most frequently affected and the lesions may show various appearances. They are rarely purely osteosclerotic, but can be cystic, lytic with sclerotic margins or have a reticular lace-like appearance [2].

Vertebral sarcoidosis is very uncommon and there are only a few cases reported in literature.

Mediastinopulmonary involvement is associated in up to $80-90 \%$ of cases [3].

Radiological and CT patterns are variable and include a lytic appearance with or without peripheral sclerosis, mixed lytic and sclerotic lesions, and purely sclerotic lesions [4].

Very few studies report MRI findings in patients with vertebral sarcoidosis. With this technique the lesions are usually multiple, ill-defined or may be well circumscribed, as seen in our patient. The signal is mostly low on $T_{1}$ weighted sequences, high on $T_{2}$ weighted sequences and enhancement is noted [3,5]. One case of vertebral sarcoid lesions showing low signal on STIR images surrounded by hyperintense margins, as seen in our patient, has been reported [4]. These rather unusual features could be related to disease activity because the signal of chronic, inactive lesions would resemble the signal of normal bone marrow, whereas active lesions would demonstrate inflammatory patterns, i.e. low signal on $T_{1}$ weighted and high signal on STIR images.

All these imaging features are non-specific. The main differential diagnoses to consider are neoplasms, including myeloma and metastases, and infectious processes, such as tuberculosis and osteomyelitis. In our case, the "rain-drop" appearance on $T_{1}$ weighted images could have been mistaken for multiple myeloma lesions.

Therefore, even in patients known to have sarcoidosis, osseous biopsy is in most cases required to affirm vertebral involvement by showing non-caseating granuloma and by excluding malignant diseases. It is possible that positron emission tomography with CT using 18-fluorodeoxyglucose would be useful to identify skeletal lesions and aid biopsy of the most active areas; however, no specific study has yet been conducted for this particular indication.

\section{Conclusion}

We report a rare case of vertebral sarcoidosis that showed well-delineated lesions, most of which appeared similar in signal to bone marrow on $T_{1}$ weighted and STIR MRI and had heterogeneous gadolinium enhancement, which corresponded to mild osteosclerotic foci on $\mathrm{CT}$. The appearance suggested inactive vertebral lesions. Even in cases of known sarcoidosis, biopsy is often required to confirm vertebral involvement and exclude other causes.

\section{References}

1. Koyama T, Ueda H, Togashi K, Umeoka S, Kataoka M, Nagai S. Radiologic manifestations of sarcoidosis in various organs. Radiographics 2004;24:87-104.

2. Fisher AJ, Gilula LA, Kyriakos M, Holzaepfel CD. MR imaging changes of lumbar vertebral sarcoidosis. AJR Am J Roentgenol 1999;173:354-6.

3. Yanny S, Toms AP, Maciver D, Barker T, Wilson A. Axial osseous sarcoidosis: rare clinical presentation and radiological findings. Clin Radiol 2010;65:167-71.

4. Poyanli A, Poyanli O, Sencer S, Akan K, Sayrak H, Acunaş B. Vertebral sarcoidosis: imaging findings. Eur Radiol 2000;10: 92-4.

5. Clarençon F, Silbermann-Hoffman O, Lebreton C, Fernandez P, Kerrou K, Marchand-Adam S, et al. Diffuse spine involvement in sarcoidosis with sternal lytic lesions: two case reports. Spine 2007;32:E594-7. 